\title{
Erratum: Universal simulation of Markovian open quantum systems [Phys. Rev. A 91, 062308 (2015)]
}

\author{
Ryan Sweke, Ilya Sinayskiy, Denis Bernard, and Francesco Petruccione
}

(Received 28 May 2017; published 14 June 2017)

DOI: 10.1103/PhysRevA.95.069904

In Sec. VI and Appendix A it was claimed that $T(t)=e^{t \mathcal{L}}$ could be approximated via a finite sequence of quantum channels $T^{(j)}\left(t^{\prime}\right)$ through the use of higher-order Suzuki-Lie-Trotter integrators of the form $\left[S_{2 k}(t / r)\right]^{r L_{1}}$ for arbitrary $k \in \mathbb{N}$. However, for $k>1$ it is in fact the case that $\left(1-4 p_{k}\right)<0$, and therefore applying the recursion rule in Eq. (A4) allows us to see that for all $k>1$ implementation of $S_{2 k}(\lambda)$ requires the simulation of multiple propagators $T^{(j)}(\tilde{t})$ with $\tilde{t}<0$ [1]. As such propagators are not quantum channels (in particular they may violate complete positivity, or even positivity) [2], it is in fact only possible to exploit first-order integrators $(k=1)$, and as such, Theorem 3 is only valid for $k=1$ as opposed to all $k \in \mathbb{N}$. In light of these considerations, Theorem 3 and the subsequent conclusions of Appendix A should be replaced with the following theorem, which is an optimized version of Theorem 3 when restricted to the case of $k=1$.

Theorem 1 . Let $1 \geqslant \epsilon>0$ be such that $(9 / 2) L_{2} m t \geqslant \epsilon$, then for

$$
r \geqslant \frac{\sqrt{2 L_{2}}(m t)^{3 / 2}}{\epsilon^{1 / 2}}
$$

we have that

$$
\left\|\exp \left(t \sum_{j=1}^{m} \mathcal{L}_{j}\right)-\left[S_{2}\left(\hat{\mathcal{L}}_{1}, \ldots, \hat{\mathcal{L}}_{m}, t / r\right)\right]^{r L_{1}}\right\|_{1 \rightarrow 1} \leqslant \epsilon,
$$

and the number of exponentials required is bounded by

$$
N_{\exp } \leqslant(2 m-1) \frac{\sqrt{2 L_{2}} L_{1}(m t)^{3 / 2}}{\epsilon^{1 / 2}} .
$$

In light of this necessary restriction to the case of $k=1$, steps (3) and (4) of the algorithm presented in Sec. VI should be modified as follows:

(3) Given $\epsilon>0$ and $t>0$, construct, as described in Appendix A, the Suzuki first-order integrator $S_{2}\left(\hat{\mathcal{L}}_{1}, \ldots, \hat{\mathcal{L}}_{m}, t / r\right)$ with

$$
r=\frac{\sqrt{2 L_{2}}(m t)^{3 / 2}}{\epsilon^{1 / 2}}
$$

where $L_{2}:=\left\|\mathcal{L}_{2}\right\|_{1 \rightarrow 1}$.

(4) Given $\rho(0)$, implement $S_{2}\left(\hat{\mathcal{L}}_{1}, \ldots, \hat{\mathcal{L}}_{m}, t / r\right)$ consecutively $r L_{1}$ times in order to recombine the linear combination in Eq. (100) through sequential implementations of $T_{k}(\tilde{t})=e^{\tilde{\tau} \mathcal{L}_{k}}$. Each implementation of $T_{k}(\tilde{t})$ is achieved via

$$
T_{k}(\tilde{t})=\mathcal{U}_{k}^{\dagger}\left[T_{A^{(k)}}(\tilde{t})\right] \mathcal{U}_{k},
$$

where $\mathcal{U}_{k}(\rho)=U^{(k)}(\rho) U^{(k) \dagger}$ and $T_{A^{(k)}}(t)=\exp \left(t \mathcal{L}_{A^{(k)}}\right)$.

As per Theorem 1 above, the modified algorithm now requires at most

$$
N=(2 m-1) \frac{\sqrt{2 L_{2}} L_{1}(m t)^{3 / 2}}{\epsilon^{1 / 2}},
$$

implementations of channels $T_{k}(\tilde{t})$ as opposed to the original claim in Eq. (105). This does not effect any of the subsequent analysis concerning the efficiency of the algorithm.

The authors would like to thank $\mathrm{T}$. Li and A. Childs for bringing the above issues to our attention.

[1] M. Suzuki, Phys. Lett. A 146, 319 (1990).

[2] H. P. Breuer and F. Petruccione, The Theory of Open Quantum Systems (Oxford University Press, New York, 2002). 